\title{
HEALTH RESEARCH, TEACHING AND PROVISION OF CARE: APPLYING A NEW APPROACH BASED ON COMPLEX SYSTEMS AND A KNOWLEDGE TRANSLATION COMPLEXITY NETWORK MODEL
}

\author{
A.H BROOK ${ }^{1,2}$, H.M LIVERSIDGE ${ }^{2}$, D WILSON ${ }^{3}$, Z JORDAN $^{4}$, G HARVEY $^{5,6}$, R.J MARSHALL \& A.L KITSON $^{5,7}$ \\ ${ }^{1}$ School of Dentistry, University of Adelaide \\ ${ }^{2}$ Institute of Dentistry, Queen Mary University of London \\ ${ }^{3}$ School of Medicine, University of Adelaide \\ ${ }^{4}$ The Joanna Briggs Institute, University of Adelaide \\ ${ }^{5}$ School of Nursing, University of Adelaide \\ ${ }^{6}$ Manchester Business School, University of Manchester \\ ${ }^{7}$ Green Templeton College, University of Oxford
}

\begin{abstract}
Despite increased emphasis on the translation of research-based knowledge into practice, studies in the U.S.A. and Australia have found that up to 50 per cent of health care delivered does not accord with evidence-based guidelines. Health research, teaching and practice have traditionally emphasised defined inputs to produce specific, linear outputs and changes in teaching and practice may suffer delays in implementation when required to overcome barriers around spheres of interest. We are exploring a new approach based on the principles of complex systems and networks. In this paper, we used a successful knowledge translation project and a case study of a natural disaster, to model the effective application of these principles to a new health knowledge translation model, the Knowledge Translation Complexity Network. Following the Indian Ocean Tsunami of 2004, there were major challenges in identifying many of the dead. Research identified that Dental Age could be used to estimate the chronological age of unidentified victims up to 20 years of age. However, at the time the existing data were insufficient for this purpose and one author (HL) undertook to lead a knowledge creation and synthesis project. The research was evaluated by peer review, published in a leading journal and was subsequently implemented into practice as an identification tool in both paper and electronic forms. Subsequently the data charts and instructions have been translated into 18 languages and are used internationally in university teaching courses as well as in disaster identifications, with feedback evaluation from users providing further refinement. In conclusion, the development of the dentitions showed the characteristics of a complex adaptive system; of emergence, self-organisation, dynamic interactions, robustness and co-evolution. Further, the Dental Atlas incorporated elements of the key sub-networks of the new Knowledge Translation Complexity Network of problem identification (PI), knowledge creation (KC), knowledge synthesis (KS), implementation (I) and evaluation (E). Investigating real-world examples in this way can both highlight key aspects for future planning and identify gaps for development.

Keywords: complex systems, complexity network, health research, knowledge translation.
\end{abstract}

\section{INTRODUCTION}

Despite increased emphasis on the translation of research-based knowledge into practice, a landmark study in the USA found that up to 50 per cent of health care delivered does not accord with evidence-based guidelines [1]. Among the reasons for this maybe that much health research, teaching and practice has been guided by a traditional, linear process paradigm that defined inputs produce specifically predictable linear outputs [2]. The assumptions underlying this paradigm may be flawed 
and so limit changes in health [2]. Rather organisations and communities are complex social systems and systems thinking is needed to understand and increase effectiveness [3].

We are exploring a new approach based on complex systems and network thinking. Our argument is that translation of new, research-derived health knowledge occurs within a complex adaptive system of many components, which can interact in a non-linear manner. The outcomes of those interactions are not fully predictable and can be greater than the sum of the inputs. The aim of this paper is to investigate a knowledge translation project in relation to a case study of a response to a natural disaster and map the effective application of these principles on a new health knowledge translation model, the Knowledge Translation Complexity Network.

\section{THE KNOWLEDGE TRANSLATION COMPLEXITY NETWORK}

This model has been developed by members of the Translational Science Task Force Team of the Faculty of Health Sciences, University of Adelaide to identify the movement of knowledge between those who generate it and those who use it [4].

We propose that Knowledge Translation is a complex network composed of five, sub-networks or 'core clusters' (problem identification, knowledge creation, knowledge synthesis, implementation and evaluation) that have multiple interactions. The pattern of these interactions varies at different tiems across the organisational systems of community, health, government, education and research. The components of this network are modelled in Fig. 1 and explained in the legend.

To optimise Knowledge Translation, participants in any of the five core clusters need to interact between the clusters. They also need to interact across the five organisational systems at the centre of the model which control funding for research, teaching and implementation. To progress the interactive nature of Knowledge Translation in the real world, it must be recognised and accounted for in health care systems.
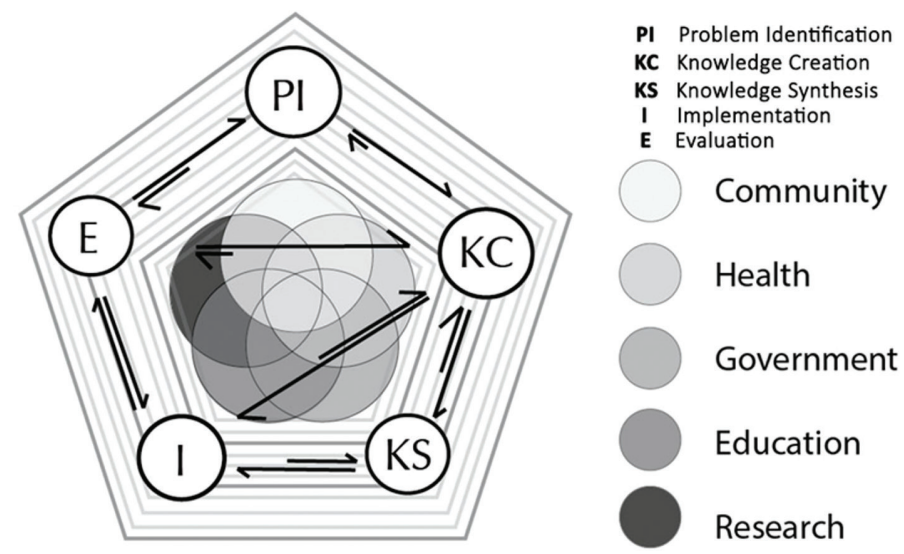

Figure 1: The KT Complexity Network Central to this knowledge translation model are five complex adaptive systems; Research, Education, Health, Government, Community. The outer five clusters of the network; problem identification (PI), knowledge creation, (KC), knowledge synthesis (KS), implementation (I), evaluation (E), function dynamically in space and time in the overall network. Each system and cluster may be weighted differently (as indicated by the size of the circles) and interact more or less frequently (as indicated by the presence/length of the arrows) depending on the needs of a given knowledge translation goal. 


\section{PROJECT OUTLINE OF THE LONDON ATLAS OF DENTAL DEVELOPMENT AND ERUPTION}

Following the Indian Ocean tsunami of 2004, there were major problems in identifying the dead. While dental age assessed from the stage of development of the teeth would be a valuable tool to estimate the chronological age of unidentified victims up to 20 years of age, at the disaster sites there was uncertainty about how this should be undertaken and what standards to use.

A leading forensic odontologist, the late Prof Jules Kieser, was a member of one of the disaster volunteer identification teams in Thailand. He contacted Dr Helen Liversidge (an author of this paper), for advice on which data and methods were the most appropriate and accurate for estimation of human age, (this represents Problem Identification (PI)). Dr Liversidge, who at that time had recently undertaken a review of three different methods for dental age, recognised the limitations of the existing databases, charts and methodologies [5]. Dr Liversidge felt unable to recommend any one method in an evidence-based way, but undertook to lead a knowledge creation study (KC) to improve the accuracy of dentition-based age identification and knowledge synthesis project (KS), aimed at developing tools and methodology that would enable implementation (I). The initial aim of KS was to develop a new Atlas that was freely available online so that Disaster Volunteer Investigation teams could access it when needed.

The initial step in knowledge creation $(\mathrm{KC})$ was evaluating $(\mathrm{E})$ the existing research through review to identifying the knowledge gaps and the limitations of the existing databases, atlases and methods of measurement. Dr Liversidge had previously undertaken extensive research both of historical skeletal material and of clinical radiographs of patients [5-7] enabling her to accelerate the rate of KS. She now also contacted national and international colleagues (across health, education and research fields) to evaluate her processes. Having evaluated (E) the existing methods and identified the gaps in the available data, the problem identification (PI), knowledge creation (KC) and knowledge synthesis (KS) questions were further refined.

At this stage, the aim was described as creating a new dental Atlas with illustrations of dentition in 1-year age categories. Discussions and evaluation with colleagues in Paediatric Dentistry, Forensic Anthropology and Palaeodemography established that a uniform age distribution was necessary with equal numbers of males and females in each age group. Following evaluation and input from a North American dental anthropologist, the median tooth stage and eruption level were selected as representing development in each age category. A postgraduate student began gathering data (KC) from dental radiographs and constructing digital illustrations (KS) of age-dependent dentition. Further evaluation (E) was sourced from a several anthropologists with considerable experience in aging skeletal and dental material and from an academic forensic odontologist. From this, the methodology was further modified.

The initial project was completed and presented for evaluation (E) to the British Association of Forensic Odontology (an organisation which spans research, education, government and health). Following evaluation (E) feedback led to additional refinements and expansion of the project. Incorporating collections of teeth from the Royal College of Surgeons of England (Health) and the Natural History Museum in London (Government and Community) allowed the categorical age range to be expanded to cover almost all the entire developing dentition from 28 weeks in utero to 23 years of age. Testing an early version of the Atlas on archaeological skeletal remains demonstrated that 1-year categories were not suitable for early development. The resultant illustrations (KS) now represented prenatal tooth formation by one month categories, the first post-natal year by 3-month categories and the remaining ages by 1-year categories (Fig. 2).

An important move at this stage was to establish world copyright to the Atlas, called The London Atlas, so that this as a knowledge synthesis tool (KS) could be made freely available without cost 


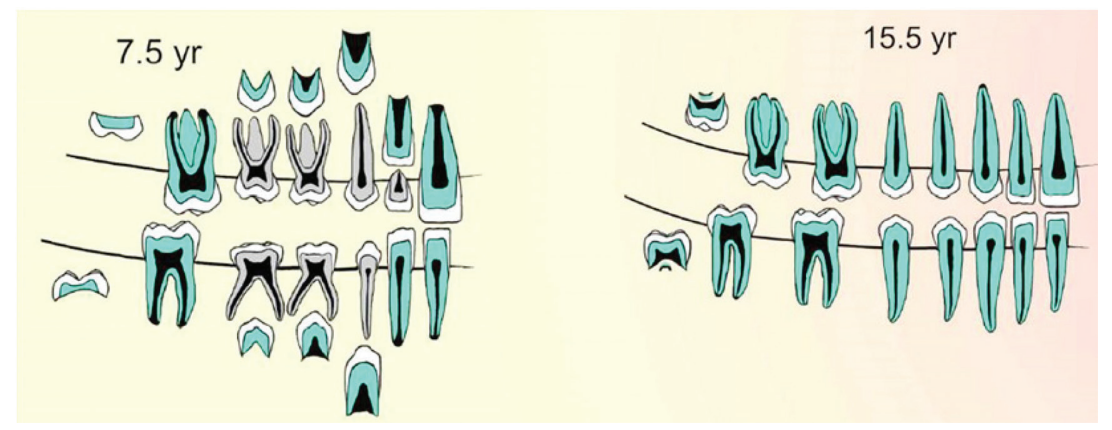

Figure 2: An example diagram from the atlas. Each tooth and eruption level represents the median tooth stage from children aged 7 and 15 , respectively.

once completed. This insightful step of obtaining world copy right precluded barriers to timely implementation (I) This was followed by an initial publication in the foremost anthropological journal, The American Journal of Physical Anthropology [8].

Next, the accuracy of The London Atlas was evaluated (E) by comparison with two existing atlases enabling the final synthesis of the new knowledge product (KS) in context. Estimated age was compared to known real age for skeletal remains or radiographs for 1,514 individuals. Excellent reproducibility was observed for all three atlases, but The London Atlas correctly estimate more cases and showed no bias while the other atlases consistently underestimated chronological age. To independently evaluate (E) the implementation (I) of the application of The London Atlas, 90 third-year dental students were randomised into three groups. Each group used one of the three atlases to estimate the radiographic age and complete a questionnaire focussed on the design, clarity, simplicity and self-explanation of the atlas. The London Atlas was preferred in all quality measures tested [9]. These findings on accuracy were also published in The American Journal of Physical Anthropology [10]. To enhance implementation (I) of the new atlas, internet and mobile app versions of the Atlas were developed. The software was sent to forensic odontologists in three countries to pilot its use (E).

Throughout the duration of the project, Dr Liversidge continued to investigate ethnic differences in tooth formation in the UK, South Africa, West and East Africa, Malaysia, Japan, Australia (Aboriginal), New Zealand (Maori), Pacific Islands and North America (Inuit and Native Americans). The timing of tooth development was similar in several groups [11]. Further work has shown that malnutrition has no significant effect [12]. Both findings underpin the validity of the use of dental age in international settings and underscore the unanticipated research and educational value that continuous evaluation (E) has provided.

The London Atlas was used more widely to estimate the ages of victims in the air crash in Libya in May 2010 and the earthquake in New Zealand in February 2011. Professor Jules Kieser commented "...my team used the (London) Atlas when we did the identification of the victims of the tragic Christchurch Earthquake. The Atlas enabled even inexperienced volunteers to understand dental aging and possibly most importantly, it was hugely useful when presentation were made to the Coroner's Board". The American Society for Forensic Odontology now list The London Atlas in their guidelines as one of the methods. In addition, The London Atlas is used in teaching forensic anthropology, forensic dentistry, dental development and archaeology. It is also used in estimating disputed age of asylum seekers and age assessment of individual archaeological skeletons. 


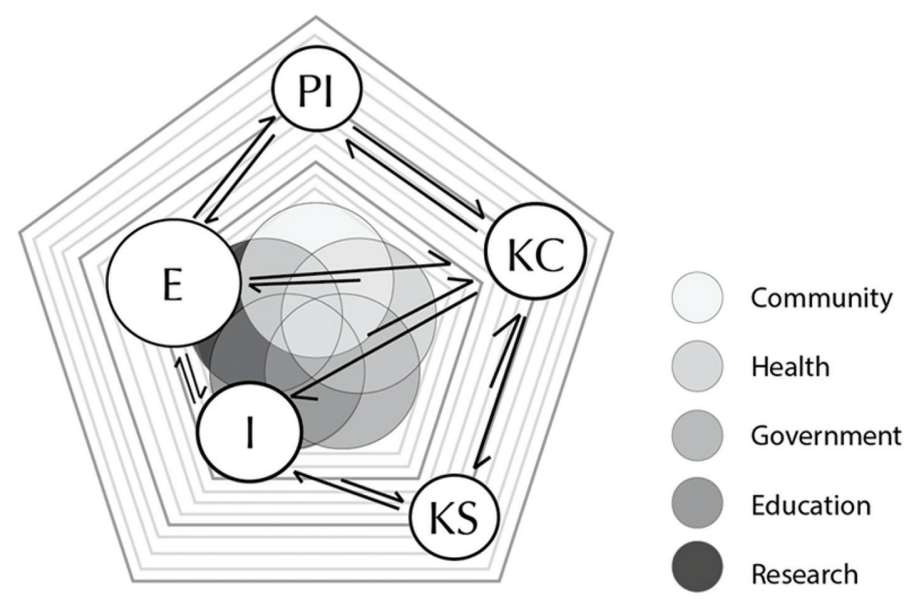

Figure 3: The London Atlas Project, identification of human age based on dentition. Problem Identification (PI) began with a goal to identify the age at death based on the dentition. Evaluation (E) of existing knowledge identified a deficiency in accuracy leading to the development of a Knowledge Creation (KC) research and interaction across the central systems of community, health, government, education and research coupled with evaluation led to an improved project goal and more defined and accurate categorical age determination. The research was developed as a tool. Knowledge Synthesis (KS). The London Atlas for chronological human dentition and further developed into an online app and a paper based illustration toolkit. Implementation of KS tools enable identification of human age. Evaluation throughout the process accelerated the rate of development.

A well-executed dissemination strategy has been key to enabling widespread use. Free access on the internet has resulted on an average 130 hits a day on the website [13]. The Atlas has been translated into 18 languages and is freely available online [14]. The app is also freely available and has been downloaded many thousands of times. A training video is also available [15].

\section{DISCUSSION}

Plotting the events of the successful project against the Knowledge Translation Complexity Network model allows both identification of the steps that enabled the highly positive outcome and also the gaps and problems that have impacted on the project. In this way, future developments for this work and for the application of the model can be highlighted.

A key factor has been the constant evaluation throughout the project that enabled its continued refinement even redefining the scope of the problem identification. The interaction with the community, represented by the end users, and the funding bodies, represented by government and universities, throughout the project. Each key sub-network of the model PI, KC, KS, I, E has been involved (Fig. 3). Problem identification occurred from the aftermath of the 2004 tsunami and was clarified by interactions with end users and professional colleagues, The Knowledge Creation built on existing knowledge that was also extended by interactive evaluation and feedback during the stages of the experimental work and development of The London Atlas and development of its distribution format. It was also farsighted to consider the implementation and obtain copyright at this 
stage to enable later open, free access to the final Atlas. It is important to highlight that repeated evaluation was embedded in the process e.g., during knowledge creation when the initial results were present to the British Association of Forensic Odontology, and the feedback was incorporated in the research. The Knowledge Synthesis, in which the research outcomes were reviewed in the light of existing data and evidence incorporated that ethnic differences and malnutrition had only limited effect on dental age, extending the application of The London Atlas as did the early establishment of world copyright.

Implementation involved the development of Internet and mobile app versions and continues with translation into different languages. Important cross links occurred with the central components of the model (Fig. 1 and 3) initially and during the project with the Saudi Arabian government funding one of the researchers (Dr AlQahtani) and Queen Mary University of London supporting implementation. The gaps were particularly limitations in funding for further applications such as an Apple version and the initial lack of involvement by the Scientific Committee of Forensic Odontology of Interpol (SCFOI). Recent discussions with SCFOI have moved this forward, and they now recommend it as one of the methods. These gaps relate to the important place of the 'government' complex within the overall network (Fig. 1 and 3) and illustrate the considerable effort required in these areas.

\section{CONCLUSIONS}

This project provided further validation for the use of dental development as a paradigm for general development. The careful and collaborative approach taken in initiating and carrying through of the project resulted in considerable impact, not only in providing a valuable tool in disaster investigation fulfilling the original need by also allowing emergence of multiple, multidisciplinary interactions. Mapping this completed project against the Knowledge Translation Complexity Network model suggests some initial validity for the model and its value in panning future work to enhance the translation of research into improved health outcomes. Using the model highlights the importance of targeted work on groups such as governments to promote the movement of new knowledge across systems.

\section{REFERENCES}

[1] Schuster, M., McGlynn, E. \& Brook, R., How good is the quality of health care in the United States? The Milbank Quarterly, 76, pp. 517-563, 1998. http://dx.doi.org/10.1111/1468-0009.00105

[2] Resnicow, K. \& Page, S.E., Embracing chaos and complexity: a quantum change for public health. American Journal Public Health, 98, pp. 1382-1389, 2008. http://dx.doi.org/10.2105/AJPH.2007.129460

[3] Sadia, R., The relationship between employee health, quality culture and organisational effectiveness: findings from the literature. International Journal of Design \& Nature and Ecodynamics, 11, pp. 1-9, 2016. http://dx.doi.org/10.2495/DNE-V11-N1-1-9

[4] Kitson, A.L., Jordan, Z., Wilson, D., Harvey, G., Marshall, R. \& Brook, A.H., Complexity, chaos and the changing discourse of knowledge translation. University of Adelaide, Faculty of Health Science, Working Paper, 2016.

[5] Liversidge, H.M., Lyons, F. \& Hector, M.P., The accuracy of three methods of age estimation using radiographic measurements of developing teeth. Forensic Science International, 131, pp. 22-29, 2003.

http://dx.doi.org/10.1016/S0379-0738(02)00373-0 
[6] Liversidge, H.M., Accuracy of age estimation from developing teeth in a population of known age (0-5.4 years). International Journal of Osteoarchaeology, 4, pp. 37-45, 1994. http://dx.doi.org/10.1002/oa.1390040107

[7] Liversidge, H.M. \& Molleson, T.I., Developing permanent tooth length as an estimate of age. Journal of Forensic Sciences, 44, pp. 917-920, 1999. http://dx.doi.org/10.1520/JFS12016J

[8] AlQahatani, S.J., Hector, M.P. \& Liversidge, H.M., Brief communication: the London Atlas of human tooth development and eruption. American Journal of Physical Anthropology, 142, pp. 481-490, 2010, available at http://www.ncbi.nlm.nih.gov/pubmed/20310064 http://dx.doi.org/10.1002/ajpa.21258

[9] AlQahtani, SJ., The London Atlas: developing an atlas of tooth development and testing its quality and performance measures. Queen Mary University of London 2012; PhD Thesis, available at https://qmro.qmul.ac.uk/xmlui/handle/123456789/8364

[10] AlQahtani, S.J., Hector, M.P. \& Liversidge, H.M., Accuracy of dental age estimation charts Schour and Massler, Ubelaker and the London Atlas. American Journal of Physical Anthropology, 154, pp. 70-78, 2014. http://dx.doi.org/10.1002/ajpa.22473

[11] Liversidge, H.M., Similarity in dental maturation in two ethnic groups of London children. Annals of Human Biology, 38, pp. 702-715, 2011. http://dx.doi.org/10.3109/03014460.2011.609565

[12] Elamin, F. \& Liversidge, H.M., Malnutrition has no effect on the timing of human tooth formation. PLoS ONE, 8, e72274, 2013. http://dx.doi.org/10.1371/journal.pone.0072274

[13] Webpage www.atlas.dentistry.qmul.ac.uk

[14] Downloadable app www.qappsonline.com/apps/atlas/

[15] Training video www.youtube.com/watch?v=FSyQvZaiXGo\&feature $=$ c4-overview \&list=UU7 JSE8G0aOmtL1oYWGueSVg 